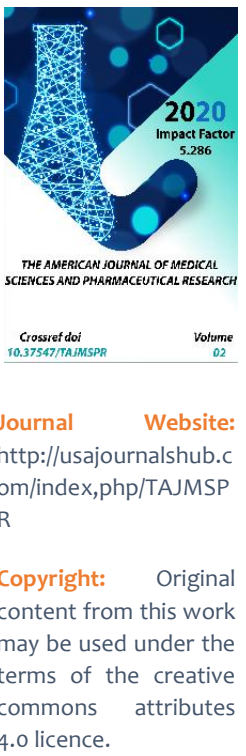

\section{Homocysteinemia As An Independent Risk Factor For The Development Of Diabetic Kidney Damage In Diabetes Mellitus}

\author{
Narbaev Abduhamid Namazovich \\ P.h.D. Assistant Department Of Internal Medicine With Endocrinology, Samarkand State \\ Medical Institute, Samarkand, Republik Of Uzbekistan \\ Togaeva Gulnora Siddiqovna \\ Assistant Department Of Internal Medicine With Endocrinology, Samarkand State Medical \\ Institute, Samarkand, Republik Of Uzbekistan \\ Kurbanova Nozima Sobirjanovna \\ Assistant Department Of Internal Medicine With Endocrinology, Samarkand State Medical \\ Institute, Samarkand, Republik Of Uzbekistan
}

\title{
ABSTRACT
}

The history of the study of hyperhomocysteinemia goes back half a century. It is interesting that the increased level of homocysteine as a factor of vascular damage was first noticed in pediatric practice. In 1969, Kilmer S. MeCully, a professor in the Department of Pathology at Harvard University, studying a rare genetic disorder manifested by homocysteinuria (increased levels of homocysteine in the urine), suggested a link between elevated serum homocysteine levels and arterial disease. Without treatment, these children usually die at an early age from myocardial infarction or cerebral strokes. During autopsy, it was found that their vessels were the same damaged and thickened as in elderly patients with cardiovascular diseases. Clinically, this disease is characterized by a marfan-like phenotype, connective tissue lesions (high growth, arachnodactyly, lens subluxation, chest deformities, heart defects), neuropsychiatric disorders, arterial thrombosis. The pathology is based on a defect in one of the metabolic enzymes of sulfur-containing methionine, mainly in the amino acid cystothionine-E-synthetase (classical tomocysteinuria). Subsequently, it was shown that moderate and mild homocysteinemia affects the vessels of various localization, leading to pathological changes in them.

\section{KEYWORDS}

Diabetes mellitus, glycemic, homocysteinemia, kidney, 


\section{INTRODUCTION}

The urgency of the problem for pediatric and adolescent diabetology is due to the fact that pre- and pubertal patients are most vulnerable to damage to the kidney vessels of a diabetic nature. in the future, these patients have a tendency to accelerated decline in renal functions, the development of chronic, renal failure. Currently, there are many theoretical prerequisites for establishing the role of homocysteinemia in the pathogenesis of diabetic kidney damage. As you know, homocysteine is an intermediate metabolic product of essential methionine, which is rich in amino acids of animal products (meat, dairy products, etc.). Like other amino acids, homocysteine is involved in the formation of proteins. It is known that the kidneys remove homocysteine from the body. Normally, the level of homocysteine in adults in the blood on an empty stomach ranges from 5 to 15 $\mu \mathrm{mol} / \mathrm{L}$. In children and adolescents, this figure is about $5 \mu \mathrm{mol} / \mathrm{l}$. The average homocysteine level in men is slightly higher than in women. A gradual increase in the level of this indicator, with age, is explained by a decrease in renal function, and higher initial values of homocysteine in men with greater muscle mass. The concentration of homocysteine in blood plasma in the range from 15 to $30 \mu \mathrm{mol} / \mathrm{L}$ indicates moderate hyperhomocysteinemia, from 30 to $100 \mu \mathrm{mol} /$ L - about intermediate, and more than 100 $\mu \mathrm{mol} / \mathrm{L}$ - about severe. The reasons for the moderate increase in plasma homocysteine concentration are known. The most significant is the hereditary predisposition to the deficiency of a number of vitamins of group B. In an experimental study conducted on rats, it was also shown that the methionine diet increases the level of homocysteine in the blood of animals. Folic acid, vitamins B6 and $\mathrm{B} 12$ are cofactors in the reverse conversion of homocysteine to methionine. In this regard, vitamin deficiency can also be the cause of hyperhomocysteinemia and associated complications. If the metabolism of homocysteine is disturbed, the excess amount is excreted from the cell into the extracellular space and then enters the bloodstream.

The negative effects of homocysteine include its direct damaging effect on the endothelium of arteries with the development of endothelial dysfunction, the ability to stimulate thrombus formation, activating the blood coagulation system, platelet aggregation, and an increase in the mitotic activity of vascular smooth muscle cells. One of the mechanisms explaining the involvement of homocysteinemia in the pathogenesis of $\mathrm{DN}$ is that an increased concentration of homocysteine can suppress the expression of heparan sulfate. Being one of the most important proteoglycans of the glomerular filtration barrier, heparan sulfate determines the charge and size selectivity of the vascular wall. With the loss of important properties of the basement membrane, an increased permeability of the glomerular filter is noted, the laboratory equivalent of which is albuminuria. An indirect argument for this effect of homocysteine is presented in a study where, when studying the culture of skin fibroblasts taken from children with cystathionine synthetase defect associated with homocysteinemia, excessive sulfation of proteoglycans in the extracellular space was revealed. The influence of homocysteinemia on the development of venous thrombosis, damage to peripheral, cerebral and cardiac vessels, the occurrence of myocardial infarction has been shown in a number of studies. Studies have established that an increase in serum homocysteine content in 
adults by $5 \mu \mathrm{mol} / \mathrm{L}$, over $10 \mu \mathrm{mol} / \mathrm{L}$ leads to an increase in the risk of developing coronary pathology by $60 \%$ in men and by $80 \%$ in women, and an increase in the risk of cerebrovascular pathology by $50 \%$ in men and women. It is known that the majority of patients on hemodialysis have an elevated level of homocysteine, and this is a risk factor for cardiovascular complications in this category of patients. In a number studies conducted over the past decade, the role of homocysteinemia in the genesis of vascular pathology is being debated. In an experimental model, insulin has been shown to influence homocysteine metabolism. Thus, in rats with streptozocin-induced diabetes not treated with insulin, activation of the enzymes of transsulfulation of homocysteine, respectively, and a decrease in its concentration were noted. while in animals receiving insulin, homocysteine levels were significantly higher. Thus, the researchers suggested that insulin therapy is a factor in increasing plasma homocysteine.

\section{CONCLUSIONS}

The study of the influence of homocysteinemia on the development of vascular complications of diabetes, including nephropathy, remains an urgent task for researchers to date.

\section{REFERENCES}

1. Algorithms of specialized medical care for patients with diabetes mellitus / ed. II .. Dedova, M.V. Shestakova. -Edit. 4th, add.- M., 2009.- p. 35-39.

2. Bondar, IA Urinary excretion of proinflammatory cytokines and transforming growth factor I in the early stages of diabetic nephropathy / IA Bondar. Bondar, V.V. Klimontov, A.P. Nadeev // Therapist, architect. 2014.-No 1. - p. 52-56.
3. Bondar, I.A. Initial changes in the kidneys in patients with type 1 diabetes mellitus / I.A. Bondar, V.V. Klimontov, A.P. Nadeev [et al.] // Problems of endocrinology. 2017, No 5. - P. 3-8.

4. Dobronravov, V.A. Modern approaches to the diagnosis and treatment of diabetic nephropathy / V.A. Dobronravov // Nephrology. -2013.-T. 7, No. 2. -p. 93-100.

5. Ignatova, M. S. Actual problems of childhood nephrology at the beginning of the XXI century / M.S. Ignatova || Pediatrics. 2007.-T. 86.- p. 67-15.

6. Kostyuchenko, G.I. Diagnostics and methods of correction of hyperhomocysteinemia in cardiological practice: a guide for doctors / G.I. Kostyuchenko, 3.S. Barkagan. M.: Nauka, 2004. - 20 p.

7. Sheiman, D.A. Kidney pathophysiology\%; per. from English. / YES. Sheiman. - M.: Binom, 2013.- p. 45.

8. American Diabetes Association. Clinical Practice Recommendations- Diagnosis and Classification of Diabetes Mellitus // Diabetes Care. - 2005 ..- Vol. 28, Suppl. 1.P. 37-42.

9. Giorgino, F. Factors associated with progression to macroalbuminuria in micro albuminuric Tire 1 diabetic patients: the EURODIAB Prospective Complications Study / F. Giorgino, L. Laviola, P. Cavallo Perin [et al.] // Diabetologia. - 2011.-Vol. 47.- P. 10201028. 